VOL. $31(1985), 5-34$.

\title{
LOCAL DEFINITIONS OF LOCAL HOMOMORPHS AND FORMATIONS OF FINITE GROUPS
}

\author{
P. Förster and $E$, Salomon
}

\begin{abstract}
It is well known that every local formation of finite soluble groups possesses three distinguished local definitions consisting of finite soluble groups: the minimal one, the full and integrated one, and the maximal one. As far as the first and the second of these are concerned, this statement remains true in the context of arbitrary finite groups. Doerk, Šemetkov, and Schmid have posed the problem of whether every local formation of finite groups has a distinguished (that is, unique) maximal local definition. In this paper a description of local formations with a unique maximal local definition is given, from which counterexamples emerge. Furthermore, a criterion for a formation function to be a local definition of a given local formation is obtained.
\end{abstract}

Consider a local formation of finite soluble groups, $F$ say. Carter, Hawkes [3] and Doerk [4] have shown that there is a unique full and integrated local definition $f^{0}$ of $F$, whereas existence of a unique minimal local definition $f_{0}$ of $F$ is evident from the mere definitions. Doerk has used the first of these to prove that the formula

$$
f^{\perp}(p)=\left\{G \in S \mid F \text { - normalisers of } G \text { belong to } f^{0}(p)\right\} \quad(p \in \mathbf{P})
$$

Received 23 July 1984.

Copyright Clearance Centre, Inc. Serial-fee code: 0004-9727/85 $\$ \mathrm{~A} 2.00+0.00$. 
defines the unique maximal local definition of $F$ (in the universe of all finite soluble groups).

Now let $F$ denote a local formation of finite (not necessarily soluble) groups. The definitions of $f_{0}$ and $f^{0}$ (see Propositions 2.1 and 1.2 below) make perfectly good sense for insoluble groups, too, and yield the corresponding results in the universe of all finite groups. Unfortunately, F-normalisers of arbitrary finite groups are not available, so Doerk's approach to the question of uniqueness of maximal local definitions cannot be generalised. (More precisely, one may indeed define F-normalisers, but these would not meet the requirements needed in the present context.) Somewhat surprisingly, in this note a more naive approach shall be shown to work (thus yielding a new proof of Doerk's result - of course, as our paper is essentially self-contained, this proof is more elaborate than the original one).

For the purpose of greater generality, and as it can be done without much additional effort, we shall employ the notion of an X-local formation as introduced in [8]. Our main result on formations suggests that greater clarity can be gained by starting with an investigation into $X$-local homomorphs, and this is confirmed in section 1 .

The reader is referred to $[2],[3],[5],[7,8]$ for definitions and basic results in the theory of homomorphs and formations. As in $[7,8], x_{0}$ denotes the class of all finite simple groups, $P \subseteq X_{0}$ is the class of all groups of prime order (and is sometimes identified with the set of all prime numbers). Throughout this paper, $X$ denotes a fixed subclass of $X_{0}$ subject to the following:

HYPOTHESIS.

$$
\left.x(x) \subseteq x \quad \text { (where } \quad x(x)=\left\{c_{p} \mid p \in \pi(X) \text { for some } X \in X\right\}\right) .
$$

As usual, $C_{p}$ is the cyclic group of order $p$. All groups shall be assumed to have finite order. Notation is (hopefully) standard.

\section{Semiformations and $x$-local homomorphs}

A semiformation is a homomorph $H$ such that $H=R_{0}^{*} H$, where $R_{0}^{*}$ is 
the closure operation on the class of all homomorphs defined by

$$
\left.R_{0}^{* K}=j G \in R_{0} K \mid Q\{G\} \cap M \subseteq K\right\} \text { for each } K \subseteq E
$$

recall that $M$ and $E$ denote the classes of all monolithic groups and of all finite groups, respectively. Equivalently, $H \subseteq E$ is a semiformation, if and only if $H=Q H$ and

$$
b(H)(=\{G \in E \backslash H \mid G / N \in H \text { whenever } 1 \neq N \unlhd G\})
$$

is contained in $M$; cf. [7], 2.1c (footnote 1 ). Of course, $R_{0}^{\prime}$-closed Schunck classes (in the sense of $[7], \S 6$ ) as well as formations are semiformations. In addition, Schunck classes are closed with respect to the closure operation $E_{\Phi}^{*}$, which is defined by

$$
E_{\Phi}^{* K}=\left\{G \in E \mid G / N \in K \text { for some } N \leq \Phi(G) \text { such that }\left(G / C_{G}(H / K)\right\}(H / K) \in K\right.
$$
whenever $H / K$ is a chief factor of $G$ below $N\}$ for each $K \subseteq E$. The following property of $E_{\Phi}^{*}$-closed semiformations shall be needed in an application of our main result.

$$
\begin{aligned}
& \text { PROPOSITION 1.1. Let } H=Q H=R_{0}^{* H}=E_{\Phi}^{* H} \text {, and put } \\
& f[H]=\{G \in E \mid(H / K) * G \in H \text { for every chief factor } H / K \text { of } G\},
\end{aligned}
$$
where

$$
(H / K) * G= \begin{cases}\left(G / C_{G}(H / K)\right)(H / K), & \text { if } H / K \text { is abelian, } \\ G / C_{G}(H / K), & \text { otherwise. }\end{cases}
$$

Then $f[H]=Q R_{0} f[H] \subseteq H$, and $F=Q R_{0} F \subseteq H$ implies $F \subseteq f[H]$; moreover, $f[H]=E_{\Phi}^{*} f[H]$.

Proof. Obviously, $f[H]$ is a formation. Assume that $f[H] \nsubseteq H$ and choose $G \in f[H] \backslash H$ of least order. Then $G \in b(H) \subseteq M$. If $S(G) \neq \Phi(G)$, we infer that $G \cong S(G) * G \in H$, a contradiction. If $S(G) \leq \Phi(G)$, then $S(G) * G \in H$ forces $G$ to be in $E_{\Phi}^{* H}=H$, another contradiction. Hence $f[H] \subseteq H$, and $F \subseteq f[H]$ for every formation $F$ oontained in $H$ follows from a well-known result of Barnes and Kegel ([1], 1.1) together with the definition of $f[H]$. Finally, $E_{\Phi}^{*}$-closure of $f[H]$ is immediate from 
$f[H] \subseteq H$

We now go on to introduce the notion of an $X$-local definition of a homomcrph in a manner which is dictated by the aims of this paper. (The most general notion of an $X$-local homomorph would require to state the definition in a way not serving our purposes, and yet would yield a class of $X$-local homomorphs only slightly larger than ours, and containing the same semiformations.)

In $\S 2$ of [8] we have indicated various ways of defining an X-Frattini subgroup $\Phi_{X}(G)$ of a finite group $G$ such that

$$
\left(\Phi\left(0_{X}(G)\right) \leq \Phi_{X}(G) \leq \Phi(G) \cap 0_{X}(G)\right)
$$

and a Gaschütz-Lubeseder-Baer theorem holds with respect to $X$-local and $X$-saturated formations $([8], 3.8)$, X-saturation being defined via $\Phi_{X}$. For the purpose of the present paper, we fix an arbitrary one of the possible definitions of $\Phi_{X}$ (subject only to the requirements pointed out in $[8])$.

An $X$-homomorph finction $k$ associates to each $X \in X(X) \cup X^{\prime}$ a homomorph $k(X)$ such that $k(X)=R_{0}^{* k}(X)$ for all $X \in X^{\prime}$; here $X^{\prime}=X_{0} \backslash X$. Given an $X$-homomorph function $k$, we let ${ }^{\mathrm{LH}} X^{(k)}$, the $x$-Local homomorph defined by $k$, be the class of all $G \in E$ such that the monolithic quotients $G / K$ of $G$ satisfy the following conditions:

(1) $G / C_{G}(S(G / K)) \in k(p)$ for each $p \in \pi(S(G / K))$, whenever $S(G / K) \ddagger \Phi_{X}(G / K)$ is an X-group (that is, a group all of whose composition factors belong to $X$ ), and

(2) $G / K \in k(E)$, whenever $S(G / K)$ is an $X^{\prime}$-group of characteristic $E$.

Clearly, ${ }^{\mathrm{LH}} \mathrm{X}^{(k)}$ is a semiformation (but is not necessarily $X$-saturated in the sense of [8], §3). $H=Q H$ is said to be an $X$-iocal homomorph provided that $H={ }^{\mathrm{LH}} \mathrm{X}(k)$ for a suitable $X$-homomorph function $k$, which then is called an $X$-Zocal definition of $H$. An $X$-homomorph function $k$ is called integrated, if $k(X) \subseteq \mathrm{LH}_{X}(k)$ for each $X \in X(X) \cup X^{\prime}$, and 
fuZZ, if $E_{p} k(p)=k(p)$ and $\mathrm{LH}_{X}(k) \subseteq E_{E^{\prime}}, k(E)$ whenever $p \in X(X)$ and $E \in X^{\prime}$, respectively $\left(E_{p}\left[E_{E^{\prime}}\right]\right.$ denotes the class of all p-groups [groups without composition factor isomorphic to $E$ ]). The class of all $X-$ homomorph functions is partially ordered by means of the following definition of $k \leq k^{\prime}$ : for each $X \in X(X) \cup X^{\prime}, k(X) \subseteq k^{\prime}(X)$.

With this terminology at hand we can formulate the obvious generalisation of the Carter-Hawkes result on certain canonical local definitions of formations of finite soluble groups, the proof of which is straightforward from the definitions together with the previous discussion and [7], 2.1 .

PROPOSITION 1.2. Let $H=\mathrm{LH}_{\mathrm{X}}(k)$. Then the unique minimal $\mathrm{X}$-local definition $k_{0}$ of $H$ is given by

$k_{0}(X)= \begin{cases}Q\left\{G / C_{G}(S(G)) \mid G \in H, S(G) \neq \Phi_{X}(G) X_{p} \text {-chief factor of } G\right\}, \\ \left\{Q, R_{0}^{*}\right\}\left\{G \in H \cap M \mid S(G) \in E_{E}\right\}, & \text { if } X=E \in X^{\prime}\end{cases}$ $\left\{x_{p}=\{X \in X \mid p \in \pi(X)\}, E_{E}\right.$ equals the class of all groups all of whose composition factors are isomorphic to $E$ ], the unique minimal full [and integrated] $X$-local definition $k_{1}$ of $H$ by

$$
k_{1}(X)=\left\{\begin{array}{l}
E_{p} k_{0}(p), \text { if } X=p \in X(X), \\
\left\{Q, R_{0}^{*}\right\}\left\{G / O_{E^{\prime}}(G) \mid G \in H\right\}, \text { if } X=E \in X^{\prime}
\end{array}\right.
$$

(where $O_{E^{\prime}}(G)$ is the largest normal subgroup of $G$ without composition factors isomorphic to $E$ ], and the conique maximal [full and] integrated $x$-local definition $k^{0}$ of $H$ is given by $k^{0}(X)=\left\{\begin{array}{lr}\{G \in H \mid G V \in H \text { for any imeducible } G F(p)[G] \text {-module } V\} \\ H, \text { if } X \in X^{\prime} . & \text { if } X=p \in X(X),\end{array}\right.$

The following observation of Doerk [5] will be applied in the proof of the main result of this section.

LEMMA 1.3. Let $H$ and $K$ be homomorphs, and put $F=h(b(K) \cap H)$. 
(Recall that $h(B)$ is the class of all groups without quotients in $B$. ) Then $F$ is the unique largest homomorph such that $F \cap H \subseteq K$; moreover, if $K \subseteq H, F \cap H=K$.

To facilitate our discussion of $X$-local homomorphs $H$ with a unique maximal $X$-local definition, we give a criterion for an $X$-homomorph function to be an $X$-local definition of $H$. The following definitions shall turn out to be crucial.

Let $H=L_{X}(k)$ with maximal integrated $X$-local definition $k^{0}$ (see Proposition 1.2). A group $G \in b_{X}(H)\left(=\left\{G \in b(H) \mid S(G) \in E_{X}\right\}\right)$ is called $X$-dense (with respect to $H$ ), if $G \in b\left(k^{0}(p)\right)$ for each $p \in \pi(S(G)$ ). Further, $b(H)$ is said to be $X$-wide, if there does not exist an $X$-dense group $G \in b_{X}(H)$. Note that a group $G \in b_{X}(H)$ with abelian socle $S(G)$ cannot possibly be $X$-dense, as otherwise $G \in E_{p} k^{0}(p) \subseteq H$, where $\{p\}=\pi(S(G)\} ;$ see Proposition 1.2. Thus in the defining condition of $X$-width of $b(H)$ we may write " $G \in b_{X}(H)$ with non-abelian socle" instead of $" G \in b_{X}(H) "$.

THEOREM 1.4. Let $H=\mathrm{LH}_{X}(k)$, and consider an X-homomorph fronction g. Then $H=\mathrm{LH}_{X}(g)$ if and only if the following two conditions hold:

(1) if $G \in b_{X}(H)$ is X-dense, then $G \notin g(p)$ for some $p \in \pi(S(G)) ;$ and

(2) $k_{0} \leq g \leq \bar{k}$, where the X-homomorph function $\bar{k}$ is defined by

$$
\bar{k}(X)=\left\{\begin{array}{l}
h\left(b\left[k^{0}(p)\right] \cap H\right), \text { if } X=p \in X(X), \\
h\left(b_{E}\left[k^{0}(E)\right]\right), \text { if } X=E \in X^{\prime} .
\end{array}\right.
$$

Proof. First suppose that $g$ is an $X$-local definition of $H$. Then every $X$-dense group $G \in b_{X}(H)$ satisfying $G \in \Pi\{g(p) \mid p \in \pi(S(G))\}$ belongs to $b(H) \cap \mathrm{LH}_{X}(g)=b(H) \cap H=\emptyset$, and so (1) holds. As for (2), $k_{0} \leq g$ is immediate from Proposition 1.2. To get that $g(p) \leq \bar{k}(p)$, 
$p \in X(X)$, we apply Lemma 1.3: it suffices to show that $g(p) \cap H \leq k^{0}(p)$, which again follows from Proposition 1.2 when $H=\mathrm{L} H_{X}\left(g_{H}\right)$ is taken into account, where $g_{H}(X)=g(X) \cap H$ for each $X \in X(X) \cup X^{\prime}$. Finally, if $g(E) \notin \bar{k}(E)$ for some $E \in X^{\prime}$, then there exists $G \in g(E) \cap b_{E}\left(k^{0}(E)\right) ; G$ can be found as a group of least order in $g(E) \backslash \bar{k}(E)$. Now $k^{0}(E)=H$, whence $G \in b_{E}(H) \subseteq M$, and from $H=L_{X}(g)$ we readily get that $G \in H$, the desired contradiction. Consequently, (2) is satisfied.

Conversely, suppose that $g$ satisfies (1) and (2). Then it remains to show that $\mathrm{LH}_{X}(g) \subseteq H$, since then $H=\mathrm{LH}_{X}\left(k_{0}\right) \subseteq \mathrm{LH}_{X}(g) \subseteq H$; here Proposition 1.2 and (2) have been applied. Consider a group $G \in \mathrm{LH}_{X}(g) \backslash H$ of least order. Then $G \in b(H)$, while $G \in b_{E}(H)$ for some $E \in X^{\prime}$ is impossible: otherwise, as $g \leq \bar{k}$ and $b_{E}(H) \subseteq M$, we should have that $G$ is in $g(E) \subseteq \bar{k}(E)=h\left(b_{E}\left[k^{0}(E)\right]\right)=h\left(b_{E}(H)\right)$. Thus $G$ is necessarily in $b_{X}(H)$. If $S(G)$ is abelian, of characteristic $p(\epsilon X(X))$, say, then we deduce that

$$
G / C_{G}(S(G)) \in g(p) \cap H \subseteq \bar{k}(p) \cap H \subseteq k^{0}(p) \text { (by Lemma 1.3), }
$$

which together with $G \in b(H)$ yields $G \in \mathrm{LH}_{\mathrm{X}}\left(k^{0}\right)=H$, a contradiction. Hence $S(G)$ is non-abelian and we have

$$
G \cong G / C_{G}(S(G)) \in g(p) \subseteq \bar{k}(p) \text { for each } p \in \pi(S(G))
$$

As $G \in b(H)$, an argument as above shows that $G / S(G) \in k^{0}(p)$ and from $k^{0}(p) \subseteq H$ we may now infer that $G \in b_{X}(H) \cap b\left(k^{0}(p)\right)$ for each $p \in \pi(S(G))$. That is to say, $G \in b_{X}(H)$ is $X$-dense, and is in each $g(p), p \in \pi(S(G))$, which contradicts (1).

COROLLARY 1.5. Let $H=\mathrm{LH}_{X}(k)$. Then $H$ has a unique maximal $x$-local definition if and only if $b(H)$ is $X$-wide; in this case $\bar{k}$ (as defined in Theorem 1.4 ) is the maximal $x$-local definition. 
Proof. In case that $b(H)$ is $X$-wide, we apply Theorem 1.4 to see that $\bar{k}$ is the unique maximal $X$-local definition of $H$ - observe that $k_{0} \leq \bar{k}$.

Conversely, suppose that $H$ possesses a unique maximal $X$-local definition $g$. Then Theorem 1.4 yields that $k_{0} \leq g \leq \bar{k}$ and $G k g(p)$ for some $p \in \pi(S(G)\}$ whenever $G \in b_{X}(H)$ is $X$-dense. We shall show that $b(H)$ is $X$-wide by proving that every $X$-dense group $G \in b_{X}(H)$ belongs to $g(p)$ for each $p \in \pi(S(G))$, and therefore cannot exist. We have to find an $X$-local definition $h_{G}^{p}$ of $H$ such that $G \in h_{G}^{p}(p)$. Such an $X$-local definition is provided by the formula

$$
h_{G}^{p}(X)=\left\{\begin{array}{l}
k^{0}(p) \cup\{G\}, \text { if } X=p, \\
k^{0}(X), \text { if } p \neq X \in X(X) \cup X^{\prime} ;
\end{array}\right.
$$

in fact, to verify that $H=\mathrm{LH}_{X}\left(h_{G}^{p}\right)$, Theorem 1.4 may be applied.

From the examples below it shall become clear that an $X$-local homomorph may or may not have a unique $X$-local definition. (Though our examples include local formations, this does not yet settle the DoerkSemetkov-Schmid problem - to do so, we have to add a few remarks; $c f$. §2.)

EXAMPLES 1.6. (a) Suppose that $X$ contains a non-abelian group $E$. Then $E$ is $X$-dense with respect to any $X$-local homomorph $H$ satisfying $E \notin H \supseteq\left\{C_{p} \mid p \in \pi(E)\right\}$; for example, take $H=N, N_{\pi(E)}$, or $S$ (nilpotent groups, nilpotent $\pi(E)$-groups, soluble groups).

(b) Let $F=N F_{0}$, where $F_{0}=Q R_{0} F_{0}$. Further, let $R_{X}$ denote the class of all $X$-groups without abelian chief factors; clearly $R_{X}=R_{X}^{2}$ is a Fitting formation. Then $F$ is an $X$-local homomorph with maximal integrated $X$-local definition $f^{0}$ given by $f^{0}(p)=E_{p} F_{0} \quad(p \in x(x))$ and $f^{0}(E)=F \quad\left(E \in X^{\prime}\right)$, and $b(F)$ is $X$-wide if and only if $R_{X} F_{0}=F_{0}$. 


\section{X-local formations}

An $X$-formation function is an $X$-homomorph function $f$ such that $f(X)=R_{0} f(X)$ for each $X \in X(X) \cup X^{\prime}$. Given an X-formation function $f$, the $X$-Zocal formation $\mathrm{LF}_{X}(f)$ defined by $f$ is the class comprising all groups $G$ such that

(1') $G / C_{G}(H / K) \in f(p)$ whenever $H / K$ is an $X_{p}$-chief factor, and

(2') $G / K \in f(E)$ whenever $S(G / K)$ is an $X^{\prime}$-chief factor of $G$ of characteristic $E$.

Observe that $\mathrm{LF}_{X}(f) \subseteq \mathrm{LH}_{X}(f)$ for every $X$-formation function $f$.

PROPOSITION 2.1. Let $f[k]$ denote an x-formation [-homomorph] fronction.

(a) $\operatorname{LH}_{X}(f)$ is a formation, and $\operatorname{LH}_{X}(f)=\mathrm{LF}_{X}(f)$.

(b) If $\mathrm{LH}_{X}(k)$ is a formation, then $\mathrm{LH}_{X}(k)=\mathrm{LF}_{X}(g)$ for a suitable $x$-formation froction $g$. The unique minimal $x$-formation function $f_{0}$ such that $\mathrm{LH}_{X}(k)=\mathrm{LF}_{X}\left(f_{0}\right)$ is the one generated by $k_{0}$ (that is, $f_{0}(X)=Q R_{0} k_{0}(X)$ for each $X \in X(X) \cup X^{\prime}$ ], the zonique maximal [full and] integrated $X$-formation fronction $f^{0}$ with the same property is the one defined in Proposition 1.2 (that is, $f^{0}=k^{0}$ ).

Proof. (a) As mentioned above $\mathrm{LF}_{X}(f) \subseteq \mathrm{LH}_{X}(f)$. Moreover, both of these classes are clearly semiformations; in fact, by [8], 3.1, the former class is a formation, and is $E_{\Phi_{X}}$-closed by $[8], 3.8$. A group of least order in $\operatorname{LH}_{X}(f) \backslash \mathrm{LFF}_{X}(f)$ is therefore necessarily in $b\left(L F X^{(f)}\right) \cap \mathrm{LH}_{X}(f) \subseteq M$, and thus cannot possibly exist.

(b) In order to verify $L H{ }_{X}(k)={ }_{L F} X^{(g)}$ for a suitable $X$-formation function $g$, it suffices to show that $k^{0}$ (as defined in Proposition 1.2) is a formation function. Since $k^{0}(E)={ }^{L H} X^{(k)}$ for any $E \in X^{\prime}$, it 
remains to deal with $k^{0}(p), p \in \chi(x)$. This, however, can be done by using a standard argument (from the proof of the Gaschütz-Lubeseder Theorem) together with the description of $k^{0}(p)$ given in Proposition 1.2. The additional assertions in (b) are clear; $c f .[8], 3.8$.

Consider an $X$-formation function $f$. The most natural candidate to serve as a unique maximal $X$-location definition of $F=L_{X}(f)$ is the X-homomorph function $\hat{f}$ - provided only that $\hat{f}$ is an $X$-formation function -, where

$$
\hat{f}(X)=\left\{\begin{array}{l}
\left\{G \in E \mid Q R_{0}\left(f^{0}(p) \cup\{G\}\right) \subseteq \bar{f}(p)\right\}, \text { if } X=p \in X(X), \\
\left\{h\left(b_{E}(F)\right), \text { if } X=E \in X^{\prime} \backslash P,\right. \\
\left\{G \in E \mid Q R_{0}\left(f^{0}(q) \cup\{G\}\right) \subseteq \bar{f}(q)\right\}, \text { if } X=q \in X^{\prime} \cap P ;
\end{array}\right.
$$

note that $h\left(b_{E}(F)\right)$ is a (saturated) formation (cf. $\left.[8], 6.3\right)$; here $\bar{f}$ is defined as in Theorem 1.4 .

THEOREM 2.2. Let $F=L_{X}(f)$ for some $X$-formation fronction $f$, and let $g$ be any $X$-formation function. Then $F=L_{X}(g)$ if and only if the following two conditions hold:

(1) if $G \in b_{X}(F)$ is $X$-dense, then $G \notin g(p)$ for some $p \in \pi(S(G))$;

(2) $f_{0} \leq g \leq \hat{f}$.

Proof. Immediate from Theorem 1.4, Proposition 2.1 and the next result.

LEMMA 2.3. Using the above notation we have

$$
\hat{f}(X)=U\left\{G=Q R_{0} G \mid G \subseteq \bar{f}(X)\right\} \text { for each } X \in X(X) \cup X^{\prime} .
$$

In particular, if $\bar{f}(X)$ contains a unique maximal formation, then the latter coincides with $\hat{f}(X)$.

Proof. By our remark above $\hat{f}(X)=Q R_{0} \hat{f}(X)$ for non-abelian $X$, and in this case the assertions hold trivially. Therefore in what follows, we may assume that $X \in \mathbf{P}$. It is clearly sufficient to verify the following statement: 


$$
Q R_{0}\{G\} \subseteq \bar{f}(X) \Rightarrow R_{0}\left(f^{D}(X) \cup Q R_{0}\{G\}\right) \subseteq \bar{f}(X)
$$

By way of contradiction, let $H \in R_{0}\left(f^{0}(X) \cup Q R_{0}\{G\}\right) \backslash \bar{f}(X)$ be of least order. Let $A \leq H^{f^{D}(X)}$ and $B \leq H^{Q R_{0}\{G\}}$ be minimal normal subgroups of $H$; note that neither $H \in f^{0}(X)$ nor $H \in Q R_{0}\{G\}$ is possible. From our choice of $H$ we have that $H / A, H / B \in \bar{f}(X)$. Furthermore, there exists $K \unlhd H$ such that $H / K \in b\left[f^{0}(p)\right] \cap F$ or $H / K \in b_{q}(F)$, according to whether $X=p \in X(X)$ or $X=q \in X^{\prime} \cap P ; c f .[7], 2.1 b$. For any choice of $A$ and $B$, subject only to the conditions stated above, we have $K \cap A=K \cap B=1$, and so $K \cap H^{f^{0}(X)}=K \cap H^{Q R_{0}\{G\}}=1$ : indeed, $\bar{f}(X) \cap F=f^{O}(X)$ has been pointed out earlier.

First assume that $X=p \in X(X)$. Then $H / K \in F$ together with $H / H^{0}(p) \in f^{\rho}(p) \subseteq F$ yields that $H$ is in $R_{0} F=F$. Consequently, by the hypothesis of $(*)$,

$$
H / H^{Q R_{0}\{G\}} \in F \cap Q R_{0}\{G\} \subseteq F \cap \bar{f}(p)=f^{O}(p)
$$

and then $H^{f^{O}(p)} \cap H^{Q R_{0}\{G\}}=I$ forces $H^{f^{O}(p)}$ to be trivial; that is, $H \in f^{\rho}(p) \subseteq \bar{f}(p)$, a contradiction.

Now suppose that $X=q \in X^{\prime} \cap \mathbf{P}$. In this case, $H / K \in b_{q}(F)$, and ${ }_{H}^{F} \leq L=S(H \bmod K)$. In addition, we know that $H^{F}=H^{f^{D}(q)}$ is minimal normal in $H$. If $B$ is as above then

$$
L=K \times B^{F}=K \times B, H^{F} \cap B=1 \text {, and } H^{F} \cong L / K \in E_{q} \text {, }
$$

whence $H / H^{Q R_{0}\{G\}} \in Q R_{0}\{G\} \subseteq \bar{f}(q)=h\left(b_{q}(F)\right)$ has a factor group belonging to $b_{q}(F)$, which is absurd.

LEMMA 2.4. Let $G$ be a formation, and let $G \in M$ with non-abelian socle be such that $G / S(G) \in G$. Then 
$Q R_{0}(G \cup\{G\})$

$$
=\left\{X \in E \mid X^{G} \leq S(X), Y \leq X^{G} \text { minimal normal in } X \Rightarrow Y * X \cong G\right\} .
$$

Proof. It is easy to see that the right hand side of the above equation defines a formation containing both $G$ and $\{G\}$. Conversely, the Jordan-Hölder Theorem may be applied to show that every group in $R_{0}(G \cup\{G\})$ has the desired structure.

Applying Lemma 2.4 enables one to deduce from Theorem 2.2 the following result in much the same way as Corollary 1.5 was obtained as a consequence of Theorem 1.4 .

COROLLARY 2.5. Let $F=L F_{X}(f)$. Then $F$ has a unique maximal $X$-Local definition (as a formation) if and only if $b(F)$ is $X$-wide and $\hat{f}(X)$ is a formation for each $X \in \mathbf{P}$; in this case $\hat{f}$ is the maximal $x$-local definition.

PROPOSITION 2.6. Let $y \subseteq X \subseteq x_{0}$ be such that both $x$ and $y$ satisfy the hypothesis stated in the introduction.

(a) If $F=L F_{X}(f)$, then $F=L_{Y}(g)$, where $g(p)=f(p)$ $(p \in \chi(y))$ and $g(E)=F \quad\left(E \in y^{\prime}\right)$.

(b) If $F=\mathrm{LF}_{X}(f)$ has a wique maximal $X$-local definition, then $F$ has a vonique maximal $y$-local definition. If, in addition, $x(x)=x(y)$, then the reverse implication is valid if, and only if, $b(F)$ is $X$-wide.

Proof. (a) is easy from the definitions.

(b) Applying Corollary 2.5, we are left to show that $\hat{g}(q)$ is a formation whenever $q \in X(X) \cap Y^{\prime}$; here $g$ is as defined in the statement of (a). We clain that

$$
\bar{g}(q)=h\left(b_{q}(F)\right) \quad\left(q \in X(X) \cap y^{\prime}\right)
$$

contains a unique maximal formation, which will give the desired result (see Lemma 2.3).

Aiming at an application of Proposition 1.1, we shall prove that $G=\bar{g}(q)$ is an $E_{\Phi}^{*}$-closed semiformation. Trivially, 


$$
b(G)=b\left(h\left(b_{q}(F)\right)\right) \subseteq b_{q}(F) \subseteq M,
$$

as $F$ is a formation. Hence $G$ is a semiformation. To get that $G=E_{\Phi}^{*} G$, we consider a group $G \in E_{\Phi}^{*} G \backslash G$ of least order. Since $Q E_{\Phi}^{*} \leq E_{\Phi}^{*} Q$ and $G=Q G$,

$$
G \in b(G) \subseteq b_{q}(F) \subseteq M
$$

and therefore the definition of $E_{\Phi}^{*}$ yields

$$
S(G) \leq \Phi(G), \quad\left(G / C_{G}(S(G))\right) S(G)=S(G) * G \in G .
$$

In fact, from $G \in b_{q}(F)$ we have $G / S(G) \in F$, which when combined with $S(G) * G \in G$ forces $S(G) * G$ to be in $F$ : otherwise $S(G) * G \in b_{q}(F) \cap G=\emptyset$. This, in turn, together with $G / S(G) \in F$ leads to $G \in F$, because $F$ is an $X$-local formation, and $S(G)$ is a $q$-group with $q \in X(X)$. We have obtained a contradiction.

For application in the next section we state a result which emerges from our proof of Proposition 2.6 (b).

COROLLARY 2.7. If $F=\left\{Q, R_{0}^{*}\right\} F$ satisfies $E_{\Phi}^{*} F \cap E_{q} F=F$ for some prime $q$, then $h\left(b_{q}(F)\right)$ is an $E_{\Phi}^{*}$-closed semiformation, and so contains a unique maximal formation.

In a similar fashion we get the following

COROLLARY 2.8. Let $F=\left\{Q, R_{0}^{*}\right\} F, H=Q R_{0} H$, and assume that $E_{\Phi}^{* F} \cap H \subseteq F$. Then $h(b(F) \cap H)$ is an $E_{\Phi}^{*}$-closed semiformation, and so contains a unique maximal formation.

\section{Some special cases}

In contrast to the condition that $b(F)$ be $X$-wide $\left(F=I_{X}(f)\right)$, the other condition from our main result corollary 2.5 - namely, that $\hat{f}(X)$ be a formation for any $X \in P$ - is not always easy to check when a specific formation $F$ is given. Imposing the closure property introduced in $\S 1$ on $f^{0}(X)$ for each $X \in \mathbf{P}$, however, removes this problem, thus providing us with a simple method to exhibit examples (which will be given 
in $\$ 4)$.

LEMMA 3.1. Let $f$ be an X-formation finction, and put $F=L F(f)$. If $f(p)$ is $E_{\Phi}^{*}$-closed for each $p \in X(X)$ and $E_{\Phi}^{*} F \cap E_{q} F=F$ for each $q \in P \cap X^{\prime}$, then $\bar{f}(X)$ is an $E_{\Phi}^{*}$-closed semiformation for each $X \in \mathbf{P}$; in particular, $\bar{f}(X)$ contains a unique largest formation, namely $\hat{f}(X)$.

Proof. First we show that $F$ is $E_{\Phi}^{*}$-closed. Assume that $G \in E_{\Phi}^{*} F F$ of least order, so $G \in b_{p}(F)$ for some $r \in \mathbb{P}$. Since $F$ is an $X$-local formation, it is clear that $r$ cannot be in $X(X)$; but then $G \in E_{\Phi}^{*} F \cap E_{r} F=F$, as $r \in P \cap X^{\prime}$. This contradiction yields that $F=E_{\Phi}^{*} F$. Now the assertions follow from Corollaries 2.7 and 2.8 together with the next observation.

REMARK 3.2. If $f$ is an $x$ formation function such that both $F=L_{X}(f)$ and $f(p)$ for some $p \in X(X)$ are $E_{\Phi}^{*}$-closed, then $f^{0}(p)$ is also $E_{\Phi}^{*}$-closed.

Proof. If $F$ and $f(p)$ are $E_{\Phi}^{*}$-closed, then so is $f(p) \cap F$ as well as $E_{p}(f(p) \cap F)=f^{0}(p)$.

As a consequence of Corollary 2.5 and Lemma 3.1 we record

COROLLARY 3.3. Let $F=L_{X}(f)=E_{\Phi}^{* F}$ be such that $f^{0}(p)$ is $E_{\Phi}^{*}$ closed for each $p \in X(X)$. Then $F$ has a mique maximal local definition if and only if $b(F)$ is X-wide.

Before discussing examples we reprove Doerk's result on formations of finite soluble groups without using the theory of F-normalisers.

THEOREM 3.4 ([4], Satz). In the coniverse $S$ of all finite soluble groups, every local (that is $x_{0}$-local, P-local) formation possesses a zonique maximal local definition.

Proof. It is clear that all of our previous results remain true in $S$, the obvious modifications of definitions, and so on, being understood. It is therefore sufficient to show that for every prime $p, \bar{f}(p)$ 
contains a unique maximal formation: recall that $X$-dense groups have nonabelian socles.

In [6], 2 , we have introduced a closure operation $S_{w}$, which is generated by the operation of taking supplements of the Fitting subgroup, and we have applied a result of Bryant, Bryce and Hartley [2] to show that formations are $\left\{Q, S_{w}\right\}$-closed. Moreover, it is clear that any class $K$ of finite groups contains a unique maximal $\left\{Q, S_{w}\right\}$-closed class (which we shall denote by $\left.K^{\{Q, S} w\right\}$. In order to prove that $\vec{f}(p)$ contains a unique maximal formation, it therefore suffices to verify the same statement with $H=(\bar{f}(p))^{\left\{Q, S_{w}\right\}}$ in place of $\bar{f}(p)$. Indeed, it suffices to show that $R_{0} H \subseteq \bar{f}(p)$, since then $Q R_{0} H \subseteq \bar{f}(p)$ implies that $Q R_{0} H \subseteq(f(p)\}^{\left\{Q, S_{w}\right\}}=H$, and this will complete the proof.

Suppose that $R_{0} H \Phi \bar{f}(p)$ and let $G \in R_{0} H \backslash \bar{f}(p)$ be of least order. Then a standard argument yields $M_{1}, \ldots, M_{n}, N \unlhd G$ such that $G / M_{i} \in H$

$$
\bigcap_{i=1}^{n} M_{i}=1, \quad G / N \in b(f(p)) \subseteq b\left(f^{0}(p)\right) \cap F
$$

Now $N \neq \Phi(G)$ : otherwise we should have that $G \in E_{\Phi} F=F$,

$$
G / M_{i} \in H \cap F \subseteq \bar{f}(p) \cap F=f^{0}(p), G \in R_{0} f^{0}(p)=f^{0}(p),
$$

which contradicts $G / N \in b\left(f^{0}(p)\right)$. Consequently, there is a chief factor $T / S$ of $G$ such that $S=N \cap \Phi(G)$ and $T \leq N$. Solubility of $G$ forces $T / S$ to be abelian, and then a well-known result of Gaschütz shows that $T \leq F(G)$. Since $T / S \$ \Phi(G / S)$, we may choose a complement $U$ of $T / S$ in $G$, that is, a subgroup $U \leq G$ satisfying $G=U T$ and $U \cap T=S$. In particular,

$$
G / M_{i}=\left(U M_{i} / M_{i}\right)\left(T M_{i} / M_{i}\right)=\left(U M_{i} / M_{i}\right)\left(F(G) M_{i} / M_{i}\right)=\left(U M_{i} / M_{i}\right) F\left(G / M_{i}\right),
$$

whence 


$$
U / U \cap M_{i} \cong U M_{i} / M_{i} \in S_{w}\left\{G / M_{i}\right\} \subseteq S_{w} H=H \quad(i=1, \ldots, n) .
$$

By choice of $G$, we see that $U \in R_{0} H \cap \bar{f}(p)$, while $G=U T=U N$ yields that $U / U \cap N \cong U N / N=G / N \in b[f(p))$, a contradiction.

In contrast to Doerk's original result, the above does not give a (more or less explicit) description of $\hat{f}(p)$, but such a description can be extracted from our proof. (Incidentally, a similar remark applies to our results in $\$ 2$.$) Nevertheless, this description does not coincide with$ the one obtained by Doerk, though the classes are necessarily the same.

Finally we would point out that it is easy to construct examples of local formations in $S$, locally defined by a formation function $f$, say, where $\bar{f}$ does not coincide with $\hat{f}$ land is not $E_{\Phi}^{*}$-closed, too - note that $E_{\Phi}^{*} \bar{f}(p)=\bar{f}(p)$ if and only if $\left.E_{\Phi}^{*} f^{0}(p)=f^{0}(p), p \in x(x)\right)$ : choose $F=N A^{0}=\left\{G \in N^{2} \mid G / F(G)\right.$ has elementary abelian Sylow subgroups $\}$, so $f^{0}(p)=E_{p} A^{0}$, whence $C_{4} \in E_{\Phi}^{*} \bar{f}(3) \backslash \bar{f}(3)$ and $C_{4} Y$ GL $(2,3)$ (direct product with Frattini subgroups amalgamated) is in $\bar{f}(3) \backslash \hat{f}(3)$.

\section{Examples and concluding remarks}

For the sake of simplicity, when dealing with particular examples, in this section we shall choose $x$ to be $x_{0} ;$ and in this case we shall omit $x$ from our notations. (Observe that " $X_{0}-l o c a l "$ actually means "local" in the sense of, for example, [3], [4].)

The most simple example of a local formation with a unique maximal local definition is given by the class $E_{\pi}$ of all $\pi$-groups, $\pi$ a set of primes. Indeed, none of our preceding results is necessary to show that

$$
\hat{f}(p)=\left\{\begin{array}{l}
E, \text { if } p \in \pi, \\
\emptyset, \text { if } p \leqslant \pi,
\end{array}\right.
$$

defines the unique maximal local definition $\hat{f}$ of $E_{\pi}$.

As an example of how to apply Corollary 3.3, we derive from Example 1.6 (b) that 
$N R=\{G \in E \mid G / F(G)$ does not have an abelian composition factor $\}$ has a unique maximal local definition: by Example $1.6(b), b(N R)$ is wide, while $E_{\Phi}^{*}\left(E_{p} R\right)=E_{p} R$ is obvious.

On the other hand, Example 1.6 (a) provides us with many examples of local formations without unique maximal local definition: for example, take $N \subseteq F \subseteq S$ - and note that $F=L F(f)$ may be chosen such that all of its full and integrated $p$-local definitions are saturated, and therefore are $E_{\Phi}^{*}$-closed.

Now consider $F_{1}=E_{p}$ and $F_{2}=N R$. We have just seen that $F_{i}$ has a unique maximal local definition $\hat{f}_{i}(i=1,2)$. However, despite the fact that $F_{1}$ is contained in $F_{2}$, the corresponding inclusion between $\hat{f}_{1}$ and $\hat{f}_{2}$ does not hold: indeed, $f_{1}(p)=E$ but every group of prime order $q \neq p$ belongs to $b\left(E_{p} R\right) \cap N R=b\left[f_{2}^{0}(p)\right] \cap F_{2}$, and is therefore not in $\hat{f}_{2}(p)$.

The latter example shows (when combined with Lemma 3.1) that in Corollary 2.5 we cannot dispense with the requirement that $b(F)$ be $X_{-}$ wide. (of course, it is already apparent from the results of $\S 1$ that this condition must not be omitted.) Far less trivial is the corresponding statement concerning the requirement that $\hat{f}(X)$ be a formation for each $X \in \mathbf{P}$. We shall give a rather complete account on an example of this type, because it involves a construction of a group, together with some formations, to yield a configuration, which is likely to serve as a sort of standard counterexample with respect to various questions in formation theory.

EXAMPLE 4.1. Let $F=N R N$ (retaining from Example 1.6 (b) the meaning of $N$ and $R$ ). By Example $1.6(\mathrm{~b})$, for each prime $p$, $f^{0}(p)=E_{p} R N$ is the full and integrated p-local definition of $F$. Then $b(F)$ is wide, yet $\hat{f}(p), p>5$, is not $R_{0}$-closed.

Proof. By Example $1.6(\mathrm{~b}), b(F)$ is wide. Therefore it remains to find a group $G \in R_{0} \hat{f}(p) \backslash \hat{f}(p), p>5$. To this purpose we construct a 
group $G \in R_{0} \hat{f}(p)$ such that $S=\mathrm{SL}(2,5) \in Q\{G\}$; since $\hat{f}(p)=\hat{Q f}(p)$, and as $S \in b\left[f^{0}(p)\right] \cap F$ - it is well known that $\Phi(S)=Z(S)$ is of order 2 and $S / Z(S) \cong A_{5}$ - this will complete the proof.

Clearly, $S$ has an irreducible module $V$ over $\mathrm{GF}(p)$ such that $C_{S}(V)=Z(S)$. Let

$$
X=S V
$$

be the semi-direct product, and let

$$
Y=S \sim_{Z(X)} X
$$

be the wreath product of $S$ and $X$ with respect to the permutation representation of $X$ on the set of cosets of $Z(X)=Z(S)$ in $X$. As usual, for any $U \leq S$,

$$
U^{*}=U \times \ldots \times U(|X / Z(X)| \text { copies of } U)
$$

shall denote the canonical subgroup of $S^{*}$, the base group of $Y$, isomorphic to a direct sum of $|X / Z(X)|$ copies of $U$. Then

$$
Z(S)^{*}=Z\left(S^{*}\right) \cong \cong_{X} \mathrm{GF}(2)[X / Z(X)],
$$

and so there exists $Z \unlhd Y$ such that

$$
Z \leq Z(S)^{*}, \quad C_{2} \cong Z(S)^{*} / Z=Z(Y / Z) \cap \Phi\left(S^{*} / Z\right) .
$$

Now we are in a position to define the above-mentioned group $G$ by putting $G=Y / Z$.

Then $S \in Q\{X\} \subseteq Q\{G\}$ is trivial.

In what follows, we use the bar convention for subgroups $U$ of $Y: \bar{U}=U Z / Z$ whenever $U \leq Y$. Employing this notation we see that

$$
A=\overline{Z(X)}, \quad B=\overline{Z(S)^{*}} \leq Z(G)=A \times B
$$

are both of order 2 and intersect trivially. Hence there exists $D \leq Z(G)$ such that $D \cap A=D \cap B=1$ and $|D|=2$. In particular, $G \in R_{0}\{G / A, G / D\}$, and so it remains to show that $\{G / A, G / D\} \subseteq \hat{f}(p)$, which (by Lemma 2.3) is equivalent to

$$
Q R_{0}\{G / A\}, Q R_{0}\{G / D\} \subseteq \bar{f}(p) .
$$


To within isomorphism, $G / A$ is a semi-direct product $X_{0} T$ satisfying $X_{0}=A_{5} V, \quad Z(T)=\Phi(T) \cong C_{2}, \quad X_{0} T / Z(T) \cong A_{5} \sim X_{0} \quad$ (a regular wreath product). In order to show that $Q R_{0}\{G / A\} \subseteq \bar{f}(p)$ we observe that

$$
A_{5}\left(A_{5}\right), V\left(A_{5}\right), T / Z(T)\left(X_{0} T / Z(T)\right), C_{2}(1)
$$

is the set of all isomorphism types of chief factors of $X_{0} T$ (together with the automorphism groups induced by $X_{0} T$ in these chief factors); of course, the same remark applies to $G / D$. We set

$$
K=\left\{A_{5}, A_{5} V, A_{5} \sim\left(A_{5} V\right), C_{2}\right\},
$$

that is, $K$ is the set of all $(H / K) *\left(X_{0} T\right)$, where $H / K$ ranges over the set of chief factors of $X_{0} T$; and we put

$$
L=\left\{L \in A_{2}^{0} D A_{p}^{O} D \mid H / K \text { chief factor of } L \Rightarrow(H / K) * L \in K\right\} \text {, }
$$

where $A_{q}^{0}$ is the class of all elementary abelian $q$-groups $(q$ any prime), and $D=D_{0}\left\{A_{5}\right\}$. Clearly, $L$ is a formation containing both

$$
L_{1}=Q R_{0}\{G / A\} \text { and } L_{2}=Q R_{0}\{G / D\} .
$$

We claim that every element of $L_{1}$ has an $N D_{0}$-projector belonging to the formation $N_{\{p, 5\}} D E_{2}$, where $D_{0}=\left\{1, A_{5}\right\}$; see [7] for a theory of Schunck classes and their projectors in finite (not necessarily soluble) groups, and note that $N D_{0}=Q\left(N D_{0}\right)$ satisfies $b\left(N D_{0}\right) \subseteq P$ and so is a Schunck class. Aiming at an application of [9], Corollary, we show that $X_{0} T \cong G / A$ has an $N D_{0}$-projector which is in $N_{\{p, 5\}} D E_{2} \cdot$ (Actually, application of [9], Corollary, requires a somewhat stronger statement, the verification of which will be left to the reader.)

Consider $X_{0} T / Z\left(X_{0} T\right) \cong A_{5} \sim X_{0}$. We shall show that this group has an ND -projector contained in $N_{\{p, 5\}} D, H_{0} / Z\left(X_{0} T\right\}$ say, and it shall become clear that $Z\left(X_{0} T\right)$, a group of order 2 , is complemented in $H_{0} \cdot$ We define $H_{0}$ by constructing its inverse image in $A_{5} \sim X_{0}$. Let $Q$ be a 
Sylow 5-subgroup of $C_{A_{5}^{*}}(V)$. Then $V \unlhd X_{0}$ yields that $X_{0} Q$ is a group isomorphic to $C_{5} \sim_{V} X_{0}$, where $\sim_{V}$ indicates that the wreath product is to be taken with respect to the permutation representation of $X_{0}$ on the set of cosets in $X_{0}$ of $V$. Since $X_{0} Q$ covers $A_{5} \sim X_{0} / A_{5}^{*}$ (with $\left.A_{5}^{*}=S\left(A_{5} \sim X_{0}\right)\right)$ being minimal normal, and as $V Q$ is $N$-maximal in $V A_{5}^{*}$, it is easy to see that $X_{0} Q \in \operatorname{Proj}_{N D_{0}}\left(A_{5} \sim X_{0}\right)$, as desired.

We have shown that $L_{1}$ is a formation contained in

$$
F_{1}=\left\{G_{1} \in E \mid \operatorname{Proj}_{N D_{0}}\left(G_{1}\right\} \cap N_{\{p, 5\}} D E_{2} \neq \emptyset\right\}
$$

Now $L_{1}=Q R_{0}\left\{X_{0} T\right\} \subseteq \bar{f}(p)$ will be a consequence of

(+) if $B \in L \cap b\left(E_{p} R N\right) \cap N R N$, then $B \cong S Y S Y \ldots Y S$ (a direct product of copies of $S=\mathrm{SL}(2,5)$ with centres amalgamated).

From $B \in L \cap N R N$ we infer that $B \in A_{2}^{0} A_{p}^{0} D:$ otherwise there should be a chief factor $B_{1} / B_{0}$ of $B$ such that $\left(B_{1} / B_{0}\right) * B \cong A_{5} \sim X_{0} \vDash N R N$. It is now clear that

$$
o_{2}(B)=Z_{\infty}(B) \in A_{2}^{0}, F(B)=o_{2}(B) \times o_{p}(B) \neq 1,
$$

and

$$
B / F(B) \cong A_{5} \times \ldots \times A_{5}
$$

note that $D \subseteq L \cap E_{p} R N$. Since $B \in b\left(E_{p} R N\right)$ is monolithic, $o_{p}(B)=1$, and so

$$
C_{2} \cong Z(B)=F(B)
$$

observe that a perfect group acting hypercentrally on some group must act trivially. Now either $Z(B) \$ \Phi(B)$, in which case $B \in M$ coincides with $c_{2} \in L \cap E_{p} R N$ - a contradiction; or $B$ is a central product of some copies of $S$ - here we rely on the well-known fact that every group $S_{0}$ 
such that $S_{0} / Z\left(S_{0}\right) \cong A_{5}$ and $2\left(S_{0}\right)=\Phi\left(S_{0}\right) \cong C_{2}$ is isomorphic to $S$.

This proves $(+)$, and we deduce that $L_{1} \subseteq \bar{f}(p)$ by assuming that $L_{1} \mid \bar{f}(p) \neq \varnothing$ and choosing $B \in L_{1} \mid \bar{f}(p)$ of least order. Then $B \in L_{I} \cap b(f(p)) \subseteq L \cap b\left(E_{p} R N\right) \cap N R N$. Hence from $(+), B \cong S Y \cdots Y S$, but then we see that every $N D_{0}$-projector of $B$ is isomorphic to $S \notin N_{\{p, 5\}} D E_{2}$. This contradicts $B \in L_{1} \subseteq F_{1}$, and so $G / A \cong X_{0} T$ generates a formation contained in $\bar{f}(p)$.

To get the same conclusion with $G / D$ in place of $G / A$, we argue similarly, applying $(+)$ to a group $B \in L_{2} \backslash \vec{f}(p)$ of least order. Then, without loss of generality, $B \in\{S, S Y S\}$; indeed, both $S$ and $S Y S$ are not in $\bar{f}(p)$, and if $B \cong S Y \dot{n}_{\dot{n}} \cdot Y S$ with $n \geq 2$, then $Q R_{0}\{B\}$ contains one of $S$ and $S$ Y S (cf. [2], \$3).

$S \notin Q R_{0}\{G / D\}$ is a consequence of $L_{2} \subseteq F_{2} \notin S$, where

$$
F_{2}=\left\{G_{2} \in E \mid \operatorname{Proj}_{E_{\Phi} D}\left(G_{2}\right\} \cap Q R_{0}\{S Y S\} \neq \emptyset\right\} ;
$$

note that (as $D$ is a formation) $E_{\Phi} D$ is a Schunck class, and $S k F_{2}$ follows from $S \in E_{\Phi} D \backslash Q R_{0}\{S$ Y $S\}$ (see [2], \$3), while $G / D \in F_{2}$ is seen from the construction of $G$.

To complete the proof, $S Y S \notin Q R_{0}\{G / D\}$ has to be verified. By the way of contradiction, let $H$ be a subdirect subgroup of $(G / D) \times \ldots \times(G / D)$ such that $H / K \cong S Y S$ for some $K \unlhd H$. Since each $p^{\prime}$-subgroup $G / D$ is contained in a Hall $p^{\prime}$-subgroup of $G / D, H$ has a Hall $p^{\prime}$-subgroup $H_{p}$, which is subdirect in ${ }^{(G / D)} p^{\prime} \times \ldots \times(G / D){ }_{n}^{\prime}$, where $(G / D)_{p}$, denotes a Hall $p^{\prime}$-subgroup of $G / D$. Clearly, $H_{p}, / H_{p}, \cap K \cong S Y S$. Hence from the construction of $G / D$ a contradiction against the conclusion of Lemna 4.2 below is easily obtained, and we are done.

LEMMA 4.2. Let $S=\operatorname{SL}(2,5)$, and let $X$ be the unique quotient of $S \sim_{Z(S)} S \quad$ (the wreath product with respect to the permutation 
representation of $S$ on the cosets of $Z(S)$ ) which is a non-split extension of the direct product of $|S / Z(S)|$ copies of $S$ with centres amalgamated by $S / Z(S)$. Then $S Y S \notin Q R_{0}\{X\}$.

Proof. Suppose that there exists a subdirect subgroup $H$ of some $X \times \ldots \times X$ with a normal subgroup $K$ such that $H / K \cong S Y S$, and choose $n$ to be minimal with respect to the property that $X \times \ldots \times X$ has such a subdirect subgroup $H$.

Recall that $X$ has a unique chief series

$$
1 \triangleleft Z \triangleleft Y \triangleleft X,
$$

where $\cdot Z \cong C_{2}, \quad Y / Z \cong A_{5} \times \underset{60}{\ldots} \times A_{5}, X / Y \cong A_{5} \cdot$ For subgroups $U$ of $X, U^{*}=U \times \underset{n}{.} \times U \leq X^{*}=X_{1} \times \ldots \times X_{n}$ shall be given a similar interpretation as previously, and we put $U_{i}=U^{*} \cap X_{i}$ (so $\left.U^{*}=U_{1} \times \ldots \times U_{n}\right)$. To begin with we have

(1) $H \cap X_{i}=z_{i}$ and $K \cap X_{i}=I(i=1, \ldots, n)$, and so $Z^{*} \leq H$.

Indeed, $H \cap X_{i} \unlhd H \prod_{j \neq i} X_{j}=X^{*} \quad$ as $H$ is subdirect in $\underset{i=1}{x} X_{i}$ ) and $H \cap X_{i} \neq 1$ (by minimality of $n$ ). Similarly, $K \cap X_{i} \unlhd X^{*}$, and thus $H \cap X_{i} / K \cap X_{i} \cong\left(H \cap X_{i}\right) K / K$ is isomorphic to a normal section of both $X$ and $S Y S$. From the structure of $X$ and $S Y S$ we see that $H \cap X_{i} / K \cap X_{i}$ is either 1 or $C_{2}$. Since $H \cap X_{i} \leq K$ is excluded by choice of $n$, we get that $H \cap X_{i} / K \cap x_{i}=S\left(x_{i}\right) / 1=z_{i} / 1$, as desired.

In what follows we shall consider $X^{*} / Z^{*}=\bar{X}^{*}$, using a bar convention for subgroups of $X^{*}$ (containing $Z^{*}$ ). Since $\bar{H}$ is subdirect in $\bar{X}^{*}$, it is readily seen that

(2) $S(\bar{H})=\bar{H} \cap S\left(\bar{X}^{*}\right)=\bar{H} \cap S(\bar{X}) *$ is subdirect in $S\left(\bar{X}_{1}\right) \times \ldots \times S\left(\bar{X}_{n}\right)$.

Further, as $\bar{H} / S(\bar{H}) \cong \bar{H} S\left(\bar{X}^{*}\right) / S\left(\bar{X}^{*}\right)$, which is subdirect in $\bar{X}^{*} / \bar{Y}^{*} \cong A_{5} \times \ldots \times A_{5}$, we have 


$$
\bar{H} / S(\bar{H})=\bar{L}_{1} / S(\bar{H}) \times \ldots \times \bar{L}_{m} / S(\bar{H}),
$$

where $\bar{L}_{i} / S(\bar{H}) \cong A_{5}$, and $\left\{\bar{L}_{i} / S(\bar{H}) \mid i=1, \ldots, m\right\}$ is the set of all minimal normal subgroups of $\bar{H} / S(\bar{H})$.

In view of (2), every minimal normal subgroup of $\bar{H}$ is isomorphic to $S\left(A_{5} \sim A_{5}\right)$ (as a group with operators). Hence combination of (2) and (3) yields

$$
S(\bar{H})=\bar{H}^{D} \quad\left(\text { where } \quad D=D_{0}\left\{A_{5}\right\}\right)
$$

Let $\Omega_{i}(i=1, \ldots, m)$ be the set of all $j \in\{1, \ldots, n\}$ such that $\bar{L}_{i}^{\pi}=\bar{X}_{j}$, where $\pi_{j}: \bar{X}_{1} \times \ldots \times \bar{X}_{n} \rightarrow \bar{X}_{j}$ denotes the projection map. Then from $\bar{X}_{j}^{\prime}=\bar{X}_{j}$ and $\left[\bar{L}_{i}, \bar{L}_{i,}\right] \leq S(\bar{H}) \leq S\left(\bar{X}^{*}\right) \quad\left(i \neq i^{\prime}\right)$ we deduce that $\{1, \ldots, n\}$ is a disjoint union of the sets $\Omega_{i} \neq \varnothing$, $i=1, \ldots, m$. Writing $S(\bar{H})=\bar{M}_{1} \times \ldots \times \bar{M}_{k}$ as a direct product of minimal normal subgroups $\bar{M}_{j}$ of $\bar{H}$, we know that $\left\{\bar{M}_{1}, \ldots, \bar{M}_{k}\right\}$ is the set of all minimal normal subgroups of $S(\bar{H})$. Since $\bar{M}_{j} * \bar{H} \cong A_{5} \sim A_{5}$, (3) and (4) show that

$$
C_{\bar{H}}\left(\bar{M}_{j}\right) \bar{L}_{i}=\bar{H} \text { for precisely one } i=i(j) \in\{1, \ldots, m\} \text {. }
$$

Observe that $i \neq i(j) \quad(i \in\{1, \ldots, m\}, j \in\{1, \ldots, k\})$ if, and only if, $\bar{L}_{i}=\bar{M}_{j} \times C_{\bar{L}_{i}}\left(\bar{M}_{j}\right)$. Let $\Omega_{i}=\{j \in\{1, \ldots, k\} \mid i=i(j)\}$ $(i=1, \ldots, m)$, so $\{1, \ldots, k\}$ is a disjoint union of the sets $\Omega_{i}$. Now put

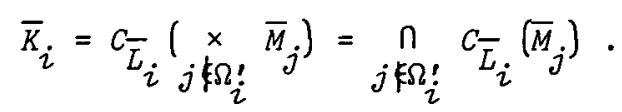

Then from $\bar{M}_{j}^{\prime}=\bar{M}_{j}$ and $\bar{L}_{i}=C_{\bar{L}_{i}}\left(\bar{M}_{j}\right) \times \bar{M}_{j}\left(j \Omega_{j}^{\prime}\right)$ one deduces the following decomposition of $\bar{L}_{i}$ :

$$
\bar{L}_{i}=\bar{K}_{i} \times\left(\underset{j \notin \Omega_{i}^{\prime}}{\times} \bar{M}_{j}\right) .
$$


Consequently, as $\bar{K}_{i} \cap S(\bar{H})=\underset{j \in \Omega_{i}^{\prime}}{\times} \bar{M}_{j}$,

$$
\bar{H}=\bar{K}_{1} \times \ldots \times \bar{K}_{m} \text {. }
$$

Furthermore,

$$
\bar{K}_{i} \text { is a subdirect subgroup of } \underset{i \in \Omega_{i}}{\times} \bar{X}_{i} \text {, }
$$

and $\bar{K}_{i} / \bar{K}_{i}^{D} \cong A_{5}$, for $\bar{X}_{\imath}=\bar{L}_{i}^{\pi^{2}}=\bar{K}_{i}^{\pi_{l}}\left(\prod_{j k \Omega_{i}^{\prime}} \bar{M}_{j}^{\pi_{l}}\right)$ yields that $\overline{\bar{K}}_{i}^{\pi^{2}}=\bar{X}_{l}$, and then $\left[\bar{K}_{i}, \bar{K}_{i}\right]=1 \quad\left(i \neq i^{\prime}\right)$ forces $\bar{K}_{i}^{\prime}$, to be contained in $2\left(\bar{x}_{2}\right)=1$.

Finally we note that the inverse image $K_{i}$ of $\bar{K}_{i}$ in $X^{*}$ may be chosen such that $z\left(\underset{Z \in \Omega_{i}}{x} X_{l}\right) \leq K_{i} \leq \underset{Z \in \Omega_{i}}{x} X_{l}$, whereas $L_{i}$ always contains $Z\left(X^{*}\right)$

With this choice we have

(5) $H=K_{1} \times \ldots \times K_{m}$, where $K_{i}$ is a subdirect subgroup of $\underset{\imath \in \Omega_{i}}{\times} X_{\imath}$ satisfying $K_{i} / K_{i}^{D} \cong A_{5}$.

Now recall that $H / K \cong S Y S$, so there are precisely two normal subgroups $A$ and $B$ of $H$ containing $K$ such that $H / A \cong A_{5} \cong H / B$. From (5) we deduce that (without loss of generality)

$$
A=K_{1}^{D} \times\left(\begin{array}{cc}
m \\
\times & K_{i=2}
\end{array}\right) \text { and } B=K_{1} \times K_{2}^{D} \times\left(\underset{i=3}{m} K_{i}\right) \text {. }
$$

Moreover, $A \cap B / K \cong C_{2}$; and $K_{1}^{D} \times K_{2}^{D} \leq K$ is impossible, for then we should have $H / K \cong C_{2} \times A_{5} \times A_{5} \neq S Y S$. Consequently, minimality of $n$ together with (5) permits us to assume that $\Omega_{3} \cup \ldots \cup \Omega_{m}=\emptyset$; that is, $H=K_{1} \times K_{2} \leq\left(\begin{array}{cc}x & X_{k}\end{array}\right) \times\left(\underset{k \in \Omega_{1}}{x} X_{k}\right)=X^{*}$. Now $K_{2} \leq A$ and $K_{1} \leq B$, but 
again $K_{Z}^{D} \ddagger K$ (for $H / K \neq A_{5} \times S$ ). This gives

$$
S \cong A / K \cong K_{2} / K_{2} \cap K \in Q\left\{K_{2}\right\}
$$

and the proof is reduced to the problem of showing that $S \neq Q\left\{K_{2}\right\}$. However, $K_{2}$ is a subdirect subgroup of some $X \times \ldots \times X$, and so the methods applied in the proof of Example 4.1, in order to show that $S \notin Q R_{0}\{G / D\}$, can be used again (with only trivial modifications being necessary) to yield $S \notin Q\left\{K_{2}\right\}$, too.

The method employed in the proof of Lemma 4.2 apparently has a wider range of applications. We hope to use it in a further study of the formation generated by an insoluble finite group (of a certain type), a problem that was left open in [2].

As a consequence of the proof of Example 4.1 we record:

COROLLARY 4.3. There exists a group $G$ with two distinct minimal normal subgroups $M_{1}, M_{2}$ such that $G \leqslant H \supseteq Q R_{0}\left\{G / M_{1}\right\} \cup Q R_{0}\left\{G / M_{2}\right\}$ for a suitable semiformation $H$.

In fact, Corollary 4.3 is weaker than what we have obtained: classes of type $h\left(b\left[f^{0}(p)\right] \cap F\right)$ are certainly not the most general semiformations.

The above example can be modified to show that $h\left(b_{q}(F)\right), F$ an $X$-local formation and $q \in X^{\prime} \cap P$, does not always contain a unique largest formation.

Put $F=E_{p} R N, p>5$ (which, by Example 4.1, is a formation), let $X$ be empty (clearly any formation is an $\phi$-local formation), put $q=2$, and take $G, A, D$ from the proof of Example 4.1. Then

$$
Q R_{0}\{G / A\} \cup Q R_{0}\{G / D\} \subseteq h\left(b_{2}(F)\right) \text {, }
$$

but

$$
G \in R_{0}\{G / A, G / D\} \backslash h\left(b_{2}(F)\right) .
$$

First of all, from the proof of Example 4.1 we know $S=\operatorname{SL}(2,5)$ is 
a quotient of $G$, and is in $b_{2}(F)$; moreover, $G \in R_{0}\{G / A, G / D\}$. Now let

$$
B_{1}=b_{2}\left(E_{p} R N\right) \cap N R N, B_{2}=b_{2}\left(E_{p} R N\right) \text { WRN; }
$$

Thus $b_{2}(F)=B_{1} \cup B_{2}$. Then $h\left(b_{2}(F)\right)=h\left(B_{1} \cup B_{2}\right)=h\left(B_{1}\right) \cap h\left(B_{2}\right)$ and we are left to verify

$$
Q R_{0}\{G / A\} \cup Q R_{0}\{G / D\} \subseteq h\left(B_{2}\right)
$$

indeed, the corresponding inclusion with $h\left(B_{1}\right)$ instead of $h\left(B_{2}\right)$ is what we have obtained in the proof of Example 4.1 , as $B_{1} \subseteq b\left(E_{p} R N\right) \cap N R N$ shows that $h\left(B_{1}\right) \supseteq h\left(b\left[E_{p} R N\right] \cap N R N\right)$.

From nilpotency of $N$ for $M, N \leq L$ such that $N / M \in N$ and $M \leq N \cap \Phi(L)$, together with $b_{2}\left(E_{p} R N\right) \subseteq M$, it is clear that all groups in $B_{2}=b\left(h\left(B_{2}\right)\right)$ are primitive groups with minimal normal 2-subgroup; in particular, $h\left(B_{2}\right)$ is a schunck class. Combining these statements we see that $h\left(B_{2}\right)$ is an $E_{\Phi}^{*}$-closed semiformation and hence (by Proposition 1.1) contains a unique maximal formation. The latter can be described as follows :

$$
f\left[h\left(B_{2}\right)\right]=\left\{G \in E \mid(H / K) * G \in h\left(B_{2}\right) \text { for every chief factor } H / K \text { of } G\right\} .
$$

Therefore it suffices to show that both $G / A$ and $G / D$ belong to this class. In view of the above characterization of $f\left[h\left(B_{2}\right)\right]$, however, this is a routine matter, which we leave to the reader. We have shown:

$E_{p} R N, p>5$, is an $\emptyset$-local formation without unique maximal $\emptyset$-local definition.

The next example will show that a local formation may have a unique maximal local definition even if its full and integrated local definition does not consist of $E_{\Phi}^{*}$-closed formations. Hence the corresponding hypothesis in Remark 3.2 is not necessary to ensure existence of a unique maximal local definition. We have to work harder, however, than in the universe of finite soluble groups (see the example in $\S 3$ ). 
EXAMPLE 4.4. Let $F_{0}=Q R_{0} F_{0}, F=N F_{0}$, and assume that

(1) $F=E_{p} F_{0}$ for some prime $p$, and

(2) $R F_{0}=F_{0}$ (that is, $b(F)$ is wide; cf. Example $1.6(\mathrm{~b})$ ).

Then $\hat{f}(p)=E, \hat{f}(q)=F_{0}$ for each prime $q \neq p$ is the unique maximal local definition of $F$; in fact $\hat{f}=\bar{f}$.

Choosing $F_{0}$ to be the class of all groups all of whose Frattini chief factors have odd order yiezds an example $F=N F_{0}$ such that (1) and (2) hold (with $p=2$ ) and, in addition, the full and integrated q-local definition of $F$, namely $E_{q} F_{0}=F_{0}$, is not $E_{\Phi}^{*}$-closed.

Proof. As we have mentioned in Example $1.6(\mathrm{~b}), f^{0}(r)=E_{p} F_{0}$ for every prime $r$. In particular, (1) ensures that $f^{D}(p)=F$, whence $b\left[f^{0}(p)\right] \cap F=\varnothing$ and $\bar{f}(p)=h(\emptyset)=E$. Another consequence of (I) is

$$
E_{q} F_{0}=f^{0}(q)=F \cap f^{0}(q)=E_{p} F_{0} \cap E_{q} F_{0}=F_{0}, q \neq p \text {. }
$$

Furthermore, $b\left[f^{0}(q)\right] \cap F=b\left(F_{0}\right) \cap N F_{0}=b\left(F_{0}\right)$ and, in view of [5], 1.1, $\bar{f}(q)=h\left(b\left[f^{0}(q)\right] \cap F\right)=h\left(b\left(F_{0}\right)\right)=F_{0}, b\left(F_{0}\right) \subseteq N F_{0}$ being a consequence of (2). Since $\bar{f}(r)$ is a formation for each prime $r$, we see that $\hat{f}=\bar{f}$ is the unique maximal local definition of $F$.

If $F_{0}=\{G \in E|N \unlhd G \Rightarrow 2||\Phi(G / N)|\}$, then clearly $E_{2}, F_{0}=F_{0}=R F_{0}$, which proves (1) plus (2). Finally, $\operatorname{SL}(2,5) \in E_{\Phi}^{*} F_{0} \backslash F_{0}$

The next (and trivial) observation provides us with a description of $\bar{f}(p)$, which might be usefur in studying further examples.

REMARK 4.5. $h(b(H) \cap F\}=\left\{G \in E \mid G / G^{F} \in H\right\}$ for homomorphs $H$ and formations $F$.

To conclude we comment on $X$-dense groups, and on $X$-local formations with $X$-wide boundaries. First we mention that the notion of an $X$-dense 
group may be defined without insisting on the use of full and integrated local definitions.

REMARK 4.6. Let $F$ be an $X$-local formation. Then $G \in b_{X}(F)$ is $X$-dense if and only if $G \in \Pi\{b[f(p)] \mid p \in \pi(S(G))\}$ for some X-local definition $f$ of $F$ satisfying $f(p) \subseteq F$ for all $p \in \pi(S(G)$ ). (By taking $X=X_{0}, F=N, g(p)=\{1\}, G=S_{5}$, however, it is seen that an $X$-dense group $G \in b_{X}(F)$ need not be in $\cap\{b(g(p)) \mid p \in \pi(S(G))\}$ for every integrated $X$-local definition $g$ of $F$.)

Finally we observe that the condition that $b(F)$ be $X$-wide can be reformulated to avoid the use of boundaries. To state such a condition we define an $X$-formation function $e$ by putting $e_{X}(p)=E_{X}, p \in X(X)$, and $e_{X}(E)=\{1\}, E \in X^{\prime}$, and give $e_{X} * f, f$ any $X$-formation function, the obvious meaning, using formation products.

REMARK 4.7. If $F=\mathrm{LF}_{X}(f)$, then $b(F)$ is $X$-wide if and only if $F=\operatorname{LF}_{X}\left(e_{X} * f\right)$.

A variation on this theme is the following

PROPOSITION 4.8. Let $F={ }^{2} X_{X}(f)$. Then $b(F)$ is $X$-wide if, and only if, the following condition holds:

$\left.E_{X} \prod_{p \in \pi(X)} f^{O}(p)\right)=\prod_{p \in \pi(X)} f^{O}(p)$ for every non-abelian $X \in X$.

Proof. $G \in b_{X}(F) \cap\left(\cap\left\{b\left[f^{0}(p)\right] \mid p \in \pi(S(G))\right\}\right)$ is equivalent to the condition that $G \in \Pi\left\{b\left[f^{0}(p)\right] \mid p \in \pi(S(G)\}\right\}$ is monolithic with nonabelian socle, the characteristic $X$ of which belongs to $X$. These groups, however, are precisely the elements of

$$
E_{X}\left(\bigcap_{p \in \pi(X)} f^{0}(p)\right) \cap b\left(\bigcap_{p \in \pi(X)} f^{0}(p)\right), \quad X \in X \backslash P .
$$

From the last result we see that every $x_{0}$-local formation $F$ such that $X(F)$ does not contain the set of primes dividing the order of a nonabelian simple group (for example, $X(F)$ the set of all odd primes, or 
$|x(F)| \leq 2$ ) has an $\mathrm{X}$-wide boundary. Utilizing this fact, it is easy to find $X_{0}$-local formations $F=L F_{X_{0}}(f)$ such that $\hat{f}$ is an $X_{0}$-formation function, but does not coincide with $\vec{f}$. For example, taking

$$
\begin{gathered}
F=L F_{X_{0}}(f)=N_{\{2,3\}} \text { with } f(2)=E_{2}=f^{0}(2), f(3)=E_{3}=f^{0}(3), \\
f(p)=\emptyset=f^{0}(p) \text { for all primes } p k\{2,3\} \text {, yields } \\
\quad \bar{f}(q)=h\left(b\left[f^{0}(q)\right] \cap F\right)=h\left(b\left[E_{q}\right] \cap N_{\{q, r\}}\right\}=h\left(c_{p}\right\},
\end{gathered}
$$

where $\{q, r\}=\{2,3\}$, and this is a schunck class, but not a formation; since the boundary of this class consists of a monolithic group, it is a semiformation, whence $\hat{f}(q)$ is the unique maximal formation contained in $\bar{f}(q)$ (see Proposition 1.1).

\section{References}

[1] D.W. Barnes and O.H. Kegel, "Gaschütz functors on finite soluble groups", Math. 2. 94 (1966), 134-142.

[2] R.M. Bryant, R.A. Bryce and B. Hartley, "The formation generated by a finite group", Bulz. Austral. Math. Soc. 2 (1970), 347-357.

[3] R.W. Carter and T.O. Hawkes, "The F-normalizers of a finite soluble group", J. Algebra 5 (1967), 175-202.

[4] K. Doerk, "Die maximale lokale Erklärung einer gesättigten Formation", Math. Z. 133 (1973), 133-135.

[5] K. Doerk, "Über Homomorphe endlicher auflösbarer Gruppen", J. AZgebra $30(1974), 12-30$.

[6] P. Förster, "Closure operations for Schunck classes and formations of finite solvable groups", Math. Proc. Cambridge Philos. Soc. 85 (1979), 253-259.

[7] P. Förster, "Projektive Klassen endlicher Gruppen. I Schunck- und Gaschützklassen", Math. 2. 186 (1984), 149-178.

[8] P. Förster, "Projektive Klassen endlicher Gruppen. II Gesättigte Formationen", Publ. Sec. Mat. Univ. Aut. Barcelona, Arch. Math. (to appear). 
[9] P. Förster, "Pull-backs of projectors in finite groups", Proc. Amer. Math. Soc. (to appear).

[10] L.A. Semetkov, Formations of finite groups (Russian) (Nauka, Moscow, 1978).

Department of Mathematics,

Monash University,

Clayton,

Victoria 3168 ,

Australia;

Fachbereich Mathematik,

J. Gutenberg-Universität,

6500 Mainz,

Federal Republic of Germany. 